\title{
THE CLINICAL SIGNIFICANCE OF THE ERYTHROCYTIC SEDIMENTATION TEST IN RHEUMATOID ARTHRITIS ${ }^{1}$
}

\author{
By WENDELL J. STAINSBY AND EDITH E. NICHOLLS
}

(From the New York Hospital, and the Department of Medicine of Cornell University Medical College, New York City)

(Received for publication June 28, 1933)

Several investigators $(1,2,3,4)$ have called attention to the increased sedimentation rate of the red blood cells in patients with rheumatoid arthritis. In order to ascertain the practical importance of this phenomenon, an extensive study was undertaken which continued for three years. During this period, routine sedimentation tests were carried out on all patients with rheumatoid arthritis on admission to the arthritis department of the Cornell Clinic. When possible, the test was repeated at varying intervals as long as a patient was under observation.

Altogether 597 cases were included in this study, and the purpose of the following paper is to present an analysis of our findings.

\section{TECHNIC}

The technic of the sedimentation test was similar in all respects to that of Rourke and Ernstene (5) and need not be described here. The method of these investigators is particularly applicable in rheumatoid arthritis because of its correction for anemia which is a common though variable factor in this disease. Heparin was used as an anticoagulant throughout the study and appeared to have no appreciable effect on the sedimentation rate. The test was recorded as the corrected sedimentation index (C.S.I.) and represents the rate of fall of the erythrocytes in millimeters per minute, after correcting for anemia. Any result up to 0.4 was considered normal. Unless otherwise stated, all sedimentation results reported refer to those at the time of the patient's first visit to the Clinic.

\section{Relation of the corrected sedimentation index to the activity of the disease}

In this report, the term "activity of the disease" refers to the severity of the infectious process. It must be recognized that this activity does not always parallel the degree of joint involvement as illustrated by "burned out" cases of arthritis in which swelling and deformity of the joints are present but the disease process is entirely quiescent.

1 This study was carried on with the technical assistance of Edith L. Ross, Edna H. Lindsay, Edith M. Kirkpatrick, and Elnora B. Carmichael. 
In patients with rheumatoid arthritis, the activity, or severity, of the disease is often difficult to determine clinically. It is roughly estimated from the amount of pain and tenderness in the joints, from the character rather than the degree of the swelling, and from the tendency of the process to migrate from joint to joint. However, temperamental factors, which vary considerably in different patients and at different times in the same individual, frequently give rise to an entirely erroneous impression concerning the activity of the disease. Weather and occupational conditions, likewise, play more or less important rôles in confusing the clinical picture. In order to determine whether the difficulties caused by these variants could be eliminated by use of the sedimentation test, the results of this method when applied to a selected group of intelligent, mentally stable, and cooperative patients were analyzed. The following protocols illustrate our findings:

Case 1. H. W., female, aged 45, admitted to the clinic March 16, 1928, gave a history of polyarthritis of six years duration. At the time of admission, pain and stiffness of the joints were slight, and the patient was pursuing her usual occupation as a typist. Physical examination revealed nothing of importance except in the joints-there was a slight but definite fusiform swelling of the fingers, with a low degree of swelling of the left knee and both ankles. A diagnosis of mild rheumatoid arthritis was made. During the succeeding three years, this patient was under our observation and throughout this period there was remarkably little change in her symptoms and physical condition. The following results of the corrected sedimentation tests, taken over part of that time, closely paralleled the clinical impression: January 1, 1931, 0.6; April 29, 1931, 0.55; September 25, 1931, 0.7; October 16, 1931, 0.6; February 29, 1932, 0.6 .

Case 2. J. Mc., male, aged 20, admitted to the clinic on January 16, 1931, gave a history of polyarthritis of two years duration and complained of considerable pain at the time of admission. Physical findings were unimportant except for the joint condition - the left hand and right knee were considerably swollen and slightly tender. During the 15 -month period that the patient was under observation, the feet, heels, shoulders, elbows, and right hand gradually became involved. He consistently complained of much pain, and tenderness was frequently noted. A diagnosis of rheumatoid arthritis was made. It was considered a very active and rapidly progressing case, without any remission during the period of observation. With this patient, the sedimentation rates closely paralleled the clinical impression, being high each time the test was made. The results of these tests were as follows: January 16, 1931, 1.7; May 1, 1931, 1.2; October 30, 1931, 1.6; November 25, 1931, 1.3; and March 16, $1932,0.75$.

Case 3 . N. DeV., male, aged 46, was admitted to the clinic August 22, 1930, with a history of a rapidly progressing polyarthritis of nine months duration. Physical examination revealed the typical fusiform swelling of the fingers, swollen knees and ankles, and stiffness of shoulders and hips. Several of the joints were tender. The remainder of the examination was irrelevant, except for the presence of a duodenal ulcer that caused no symptoms while the patient was under observation. He showed no improvement while attending the clinic, and was admitted to the hospital January 2, 1931. A corrected sedi- 
mentation test, carried out on December 29, 1930, a few days before admission, was 1.23. With rest in bed the patient made rapid progress toward recovery, and was discharged from the hospital on February 28, 1931, as a cured case. At that time he was entirely free from symptoms. There was, however, a small amount of swelling still remaining in the fingers and wrists, but this was considered as due to the scarring resulting from the disease, rather than as representing an active process. The corrected sedimentation tests during his stay in the hospital closely paralleled his clinical improvement, being 1.2 on January 2, 1931; 0.7 on February 7, 1931; and 0.4 on February 25, 1931-the figure recognized as normal in a corrected sedimentation test. Following his discharge from the hospital, the patient returned to work. On resuming his occupation, there was a marked return of the arthritic process. On May 19, 1931, his clinical condition approximated that on admission to the hospital four months previously, and his corrected sedimentation test was 1.6. During the succeeding year, this case was followed in the out-patient department, but there was no appreciable change noted. On April 29, 1932, when last seen, his arthritis was unimproved and his corrected sedimentation test was 1.9.

Case 4. M. O., female, aged 40, was admitted to the clinic March 14, 1930, with a three year history of pain and intermittent swelling in fingers and ankles. Symptoms had never been severe, and she had been able to continue her household activities. Physical findings were unimportant except in respect to the joints. There was questionable swelling of the proximal interphalangeal joints and slight puffiness around the ankles. A diagnosis of mild rheumatoid arthritis was made. During the succeeding year she was observed in the clinic at irregular intervals, with little if any evidence of arthritis. On February 3, 1931, the date of her first test, the corrected sedimentation index was 0.2 .

Beginning in March, 1931, her arthritis gradually grew worse, and the fingers took on a marked fusiform swelling. In succession, the knees, wrists, elbows, and shoulders became swollen and painful. There were aching pains in practically every joint in the body. The sedimentation test coincided perfectly with the change in her clinical condition, the index being 0.9 on April 15, 1931, and 1.3 on April 23, 1931. On May 11, 1931, she again attended the clinic. At that time she was barely able to walk and her sedimentation index was 1.3. On May 23 of the same year, she made her last visit to the clinic and her index at that time was 1.1. Following this observation, she reported by telephone that she was unable to leave her bed.

Case 5. J. M., male, aged 24, admitted to the clinic on December 20,1930, gave a history of a rapidly progressing polyarthritis of ten weeks duration. Physical examination showed little of importance except for the joint condition. There was fusiform swelling of several fingers, with swollen and tender knees and ankles. A diagnosis of rheumatoid arthritis was made, and the condition was so severe that the patient was admitted immediately to the hospital. The sedimentation index on December 20,1930, was 2.0. On the day of admission, the patient's temperature was $99.5^{\circ} \mathrm{F}$., and was normal thereafter. With rest in bed and hospital care the arthritis rapidly improved from the day of admission. On February 13, 1931, the swelling had completely disappeared, and on February 25, twelve days later, the patient was discharged as free from signs and symptoms of the disease. The clinical change was correctly and immediately registered in his sedimentation tests. On December 29, 1930, the index was 0.65 ; January 13, 1931, it was 0.4 ; on February 7 and 25 of the same year it was 0.1 and 0.2 , respectively. 
The first protocol is that of a patient running a mild course of arthritis throughout observation. Her sedimentation indices recorded at varying intervals during this time were remarkably consistent with the clinical findings, being slightly above normal each time the test was made. A similar relation between the sedimentation rate and the clinical findings was revealed in the second protocol, although the second patient had a severe process, and his sedimentation indices were consistently high.

Protocols 3, 4, and 5 are of patients whose clinical picture varied markedly during observation. The third patient had a complete remission; the fourth developed a severe form of the disease from a very mild one, while the fifth recovered completely from a very active arthritis. The sedimentation indices in each of these cases correctly and immediately registered the changes in the patient's physical condition.

In the other patients of our series the relationship between the clinical picture and the sedimentation rate is similar to that in the five protocols given. Regardless of the fundamental factors underlying the sedimentation phenomenon, it is apparent that the erythrocytic sedimentation index parallels closely the severity, or activity, of the arthritic process.

\section{Relation of corrected sedimentation index to the degree of joint involvement}

In the preceding section of our report, it was shown that the corrected sedimentation index is directly related to the activity, or severity, of the arthritic process. In the present section, a comparison of this index with the degree of joint involvement is considered. A differentiation between the activity, or severity, of the disease and the degree of joint involvement must be made, as it is recognized clinically that some patients suffering from a severe form of the disease have considerable pain, malaise, and even disability, with little change in the joints that may be detected by physical examination, whereas a patient may have marked swelling of the joints with deformity and ankylosis without the symptoms named above.

In Table 1, the 597 patients studied are tabulated according to degree

TABLE 1

Relation of corrected sedimentation index to degree of joint involvement

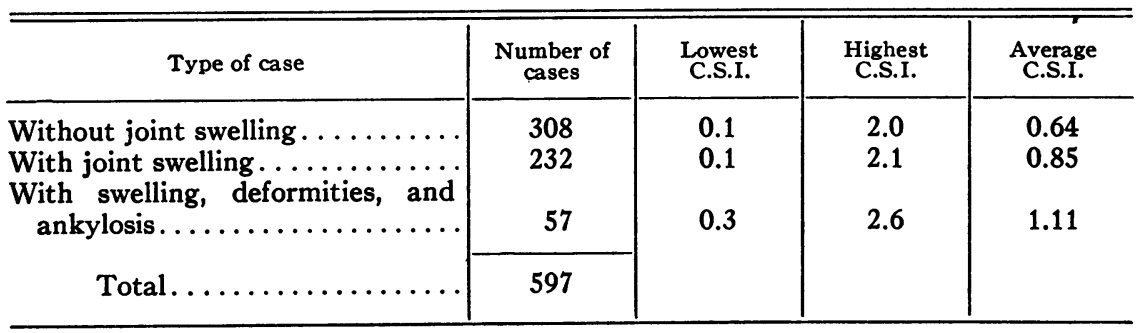


of joint involvement. Three hundred and eight of these had no appreciable joint changes at the time of examination; their sedimentation indices ranged from 0.1 to 2.0, with an average of 0.64. Two hundred and thirty-two of them had joint swelling without ankylosis, or deformities other than swelling; their sedimentation indices varied from 0.1 to 2.1 , with an average of 0.85 . Fifty-seven of them were in the more advanced stages of the disease with deformities such as ulnar deviation and ankylosis; the sedimentation indices of this group ranged from 0.3 to 2.6, with an average of 1.11 .

From these results it is evident that, on the average, the greater the degree of swelling, deformity, and ankylosis, the higher the sedimentation rate, and, therefore, the greater the activity, or severity, of the disease, but as far as the individual patient is concerned, this deduction cannot always be made.

\section{Relation of the corrected sedimentation index to the age of the patient}

In Table 2, the corrected sedimentation indices of the 597 cases are arranged according to the age of the patient. In this comparison, an interesting though unexpected relation was revealed. In patients in the third and fourth decades of life, a similar average sedimentation index was obtained but in those $\mathbf{4 0}$ years of age or over the average sedimentation rate showed a gradual increase all the. way to the last group composed of patients 60 years of age or older.

TABLE 2

Relation of corrected sedimentation index to age of patient

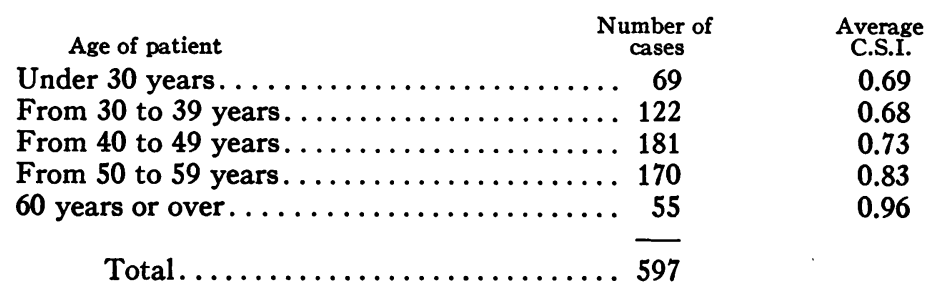

The explanation of this gradual increase in the sedimentation rate with advancing years is not altogether clear. In the first place, it would seem probable that as patients grow older they tend to develop disease conditions other than arthritis which also affect the sedimentation rate. Our records indicate, however, that this factor plays no important rôle in the phenomenon, as no appreciable increase in disease unrelated to arthritis was detected in the older groups of our series. In the second place, it was observed that each succeeding decade is augmented by an increasing proportion of patients with severe arthritis who failed to recover from the disease earlier in life, as evidenced by an increase of the average disease duration with each succeeding age period. It seems 
reasonable to conclude, therefore, that the increase in the average sedimentation rate with each succeeding age period is due primarily to the increase in the number of patients with severe arthritis, although other factors may play minor rôles in the results.

Relation of corrected sedimentation index to season of year

Clinically it is generally recognized that in temperate climates patients with rheumatoid arthritis are usually at their worst in cold weather and at their best in hot weather. In order to determine whether this impression is confirmed by the sedimentation test, the indices of patients followed through the various seasons were analyzed. All patients on whom tests had been made in at least three seasons were included. With those having more than one test during any one seasonal period, the average was considered as that patient's index for that season. In the study, "Winter" covers the period from January 1 to May 31 ; "Spring" from April 1 to June 30; "Summer" from July 1 to September 30; and "Autumn" from October 1 to December 31.

TABLE 3

Relation of corrected sedimentation index to season of year

\begin{tabular}{|c|c|}
\hline $\begin{array}{l}\text { Number of } \\
\text { indices }\end{array}$ & $\begin{array}{c}\text { Average } \\
\text { C.S.I. }\end{array}$ \\
\hline Winter............ & 0.865 \\
\hline 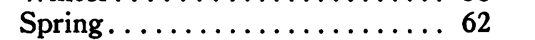 & 0.840 \\
\hline Summer. . . . . . . . . . . 38 & 0.799 \\
\hline Autumn $\ldots \ldots \ldots \ldots \ldots \ldots \ldots 55$ & 0.846 \\
\hline
\end{tabular}

Altogether 69 patients were studied for seasonal variation. On these 69 patients, 58 tests were recorded in the winter; 62 , in the spring; 38 , in the summer; and 55, in the autumn. In Table 3, the average corrected sedimentation index for each of these recorded groups is presented. The highest figure occurred in winter, and the lowest, in summer, while spring and autumn gave intermediate results. The number of cases studied and the differences between the seasonal averages were too small to warrant definite conclusions. However, it is strongly suggestive that seasonal variations in the average sedimentation indices do occur, and as this test is an index of the activity, or severity, of the arthritis, that patients, on the average, are worse in the winter and better in the summer.

\section{Relation of the corrected sedimentation index to the duration of the disease}

The records of the patients under observation were studied again to discover whether any relationship existed between the corrected sedimentation index and the duration of the disease. In the individual records little such relationship was found-some patients having had the disease for only a few weeks gave high sedimentation rates, while others having had it for many years gave a low sedimentation rate, and 
vice versa. In studying the average rates of sedimentation for various disease-duration periods, however, it was found that there was a distinct tendency for these rates to increase as the duration of the disease increased (Table 4). From these results we conclude that patients having rheumatoid arthritis for a long period tend to have a more severe form of the disease than those having it for a short period, but that great individual variation exists.

TABLE 4

Relation of corrected sedimentation index to duration of disease

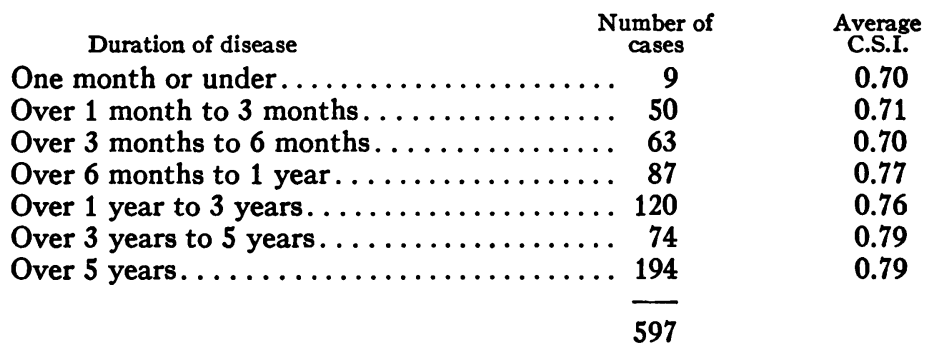

Relation of the corrected sedimentation index to the streptococcus agglutination test

In previous articles $(6,7)$, the authors showed that a high percentage of patients with rheumatoid arthritis gave a strong agglutination reaction with a biologically specific type of hemolytic streptococcus which, for convenience, they designated as "typical strain." This reaction appeared to be a true immunological response to a bacterial invader. The question naturally arises as to what relationship, if any, exists between this agglutination reaction and the erythrocytic sedimentation rate. An examination of the records of the cases in our series indicated that there was none whatever. In patients with rheumatoid arthritis, the agglutinins are slow in developing and do not reach their maximum titer until about 6 months, on an average, has elapsed. Following recovery of the patients, the agglutinins gradually diminish, but do not disappear, usually, until the patient has been free from the disease for several months, and sometimes years. The sedimentation rate, on the other hand, is directly related to the activity, or severity, of the disease process, and changes directly with variations in this activity. The difference in these two tests may be appreciated readily from a study of Figure 1, which is a graphic record of the agglutination and sedimentation tests of Case 5, previously described, and is typical of other similar records.

\section{DISCUSSION AND SUMMARY}

The erythrocytic sedimentation test was performed on five hundred and ninety-seven patients with rheumatoid arthritis in order to determine its significance in this disease. 


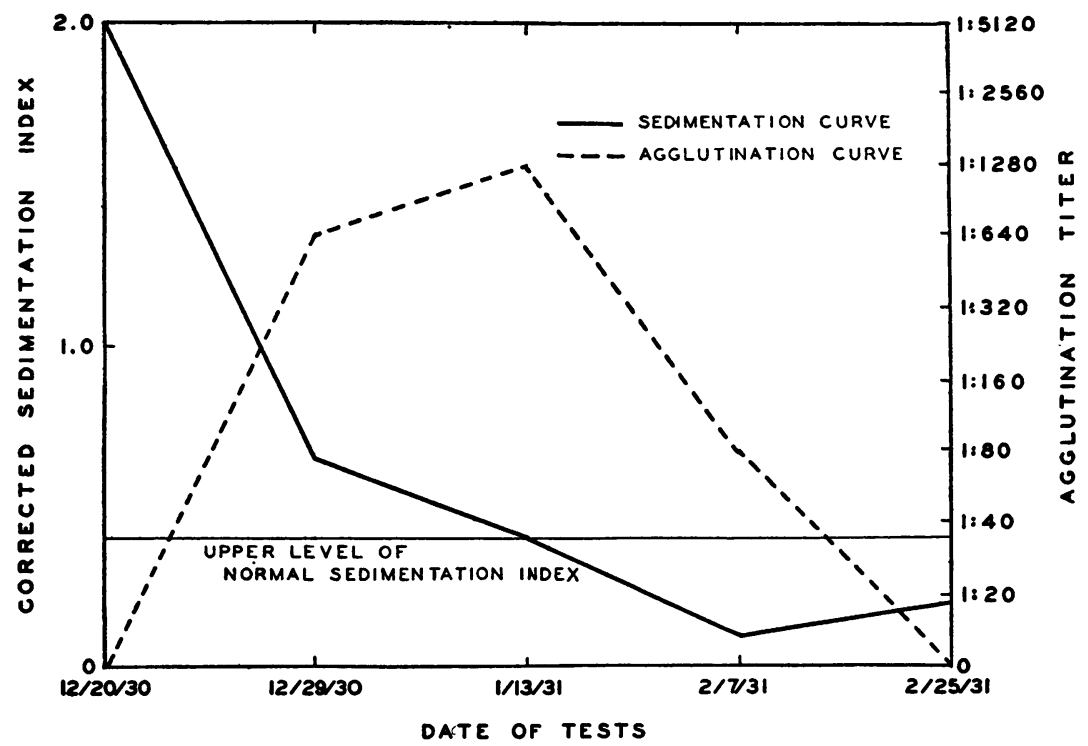

Fig. 1. A Comparison of the Results of the Sedimentation and Agglutination Tests of Case 5

The results of the investigation indicate that the corrected sedimentation index is a reliable criterion of the activity, or severity, of the arthritic process at the time of testing, and that any fundamental change in the clinical condition produces immediate corresponding change in the sedimentation rate.

Patients with a greater degree of joint involvement and a longer disease-duration have higher sedimentation rates, on the average, than those with less joint involvement and shorter disease-duration. Considerable variation occurs, however, in individual cases.

The observation that the average sedimentation rate progressively increases with advancing age periods is of interest. This phenomenon appears to be due primarily to the increasing accumulation of patients with severe arthritis in whom the disease began at some earlier age.

From a study of seasonal variations in the sedimentation rate over a long period, suggestive evidence was deduced that, on the average, the rate was higher in winter than in summer, while spring and autumn occupied intermediate positions. Further work is necessary in order to confirm this impression.

No relationship was found between the sedimentation rate and the streptococcus agglutination reaction.

With the above information at hand, it seems justifiable to discuss the practical importance of this test. In the past, physicians treating arthritis have been greatly handicapped by lack of means for estimating 
the results of their therapy. The sedimentation test appears to supply this widely felt need, as it is a reliable measuring rod of the activity, or severity, of the arthritic process. By repeating this test at regular intervals, the progress of the patient may be determined.

Rheumatoid arthritis has attracted more than its share of ill-conceived treatments. The sedimentation test provides a ready aid for correctly estimating the value of such procedures.

\section{BIBLIOGRAPHY}

1. Kahlmeter, G., Klin. Wchnschr., 1926, v, 889. Über die Bedeutung der Fåhreusschen senkungsreaktion bei akuten und chronischen Arthritiden.

2. Dawson, M. H., Sia, R. H. P., and Boots, R. H., J. Lab. and Clin. Med., 1930, xv, 1065. The Differential Diagnosis of Rheumatoid and Ostearthritis: The Sedimentation Reaction and Its Value.

3. Weiss, Arthur, Am. J. M. Sc., 1931, clxxxi, 379. The Prognostic Value of the Sedimentation Rate in Arthritis. A Modification of the Technique.

4. Oppel, T. W., Myers, W. K., and Keefer, C. S., J. Clin. Invest., 1933, xii, 291. The Sedimentation Rate of the Red Blood Cells in Various Types of Arthritis.

5. Rourke, M. D., and Ernstene, A. Carlton, J. Clin. Invest., 1930, viii, 545. A Method for Correcting the Erythrocyte Sedimentation Rate for Variations in the Cell Volume Percentage of Blood.

6. Nicholls, E. E., and Stainsby, W. J., J. Clin. Invest., 1931, x, 323. Streptococcal Agglutinins in Chronic Infectious Arthritis.

7. Nicholls, E. E., and Stainsby, W. J., J. Clin. Invest., 1933, xii, 505. Further Studies on the Agglutination Reaction in Chronic Arthritis. 